\title{
Assessment of left ventricular volumes using simplified 3-D echocardiography and computed tomography - a phantom and clinical study
}

\author{
Mattias Mårtensson*1, Reidar Winter², Kerstin Cederlund³, \\ Jonaz Ripsweden ${ }^{3}$, Habib Mir-Akbari ${ }^{4}$, Jacek Nowak² and Lars-Åke Brodin ${ }^{1}$
}

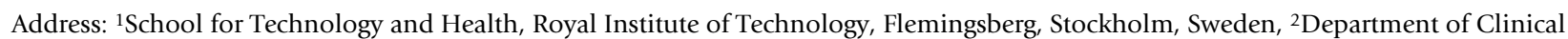
Physiology, Karolinska University Hospital in Huddinge, Stockholm, Sweden, ${ }^{3}$ Department of Radiology, Karolinska Institutet, Stockholm, Sweden and ${ }^{4}$ Department of Cardiology, Karolinska University Hospital in Huddinge, Stockholm, Sweden

Email: Mattias Mårtensson* - mmar@kth.se; Reidar Winter - reidar.winter@karolinska.se; Kerstin Cederlund - kerstin.cederlund@karolinska.se; Jonaz Ripsweden - jonaz.ripsweden@karolinska.se; Habib Mir-Akbari - habib.mir-akbari@karolinska.se; Jacek Nowak - Jacek.Nowak@ki.se; Lars-Åke Brodin - Lars-Ake.Brodin@syd.kth.se

* Corresponding author

Published: 4 June 2008

Cardiovascular Ultrasound 2008, 6:26 doi:10.1186/1476-7/20-6-26
Received: 28 April 2008

Accepted: 4 June 2008

This article is available from: http://www.cardiovascularultrasound.com/content/6/1/26

(C) 2008 Mårtensson et al; licensee BioMed Central Ltd.

This is an Open Access article distributed under the terms of the Creative Commons Attribution License (http://creativecommons.org/licenses/by/2.0), which permits unrestricted use, distribution, and reproduction in any medium, provided the original work is properly cited.

\begin{abstract}
Objectives: To compare the accuracy of simplified 3-dimensional (3-D) echocardiography vs. multi-slice computed tomography (MSCT) software for the quantification of left ventricular (LV) volumes.

Design: Three-D echocardiography (3-planes approach) and MSCT-CardIQ software were calibrated by measuring known volumes of 10 phantoms designed to closely mimic bloodendocardium interface. Subsequently, LV volumes were measured with both the methods in 9 patients referred routinely for coronary angiography and the agreement between the measurements was evaluated.
\end{abstract}

Results: Simplified 3D-echocardiography provided higher degree of agreement between the measured and true phantom volumes (mean difference $0 \pm \mathrm{I} \mathrm{ml}$, variation range +4 to $-4 \mathrm{ml}$ ) than MSCT software (mean difference $6 \pm 5 \mathrm{ml}$; variation range +22 to $-10 \mathrm{ml}$ ). The agreement between LV measurements in the patients was considerably poorer, with significantly larger volumes produced by MSCT (mean difference $-23 \pm 40 \mathrm{ml}$, variation between +93 and $-138 \mathrm{ml}$ ).

Conclusion: Simplified 3-D echocardiography provides more accurate assessment of phantom volumes than MSCT-CardIQ software. The discrepancy between the results of LV measurements with the two methods is even greater and does not warrant their interchangeable diagnostic use.

\section{Background}

Echocardiography and x-ray based computed tomography provides possibilities of detailed evaluation of cardiac morphology and function and the introduction of these techniques constitutes without any doubt an important landmark in the history of diagnostic cardiology. Today, both the methods not only form a backbone of diagnostic cardiac procedures but also are much appreciated as sensitive research instruments. Recently, the diagnostic 
capacity of echocardiography has been further improved by the addition of 3-dimensional cardiac imaging.

An important link in the process of cardiac diagnostics is accurate estimation of left ventricular volume. In this respect, MSCT has been shown to be both feasible and accurate when compared with left ventriculography [1], magnetic resonance techniques [2-4], and 2-dimensional echocardiography [4-7]. At the same time, the diagnostic performance of 3-D echocardiography has been demonstrated to be superior to that provided by 2-dimensional echocardiographic imaging [8-13] and a strong correlation was observed between the results obtained with 3-D echocardiographic technique and magnetic resonance imaging $[9,11,14-17]$.

However, the results of a recently published study indicate that even though both 3-D echocardiography and MSCT measurements correlate highly with magnetic resonance imaging, 3-D echocardiography compares more favourably in this respect than MSCT that tend to overestimate the magnetic resonance values [17]. Therefore, the aim of this study was to evaluate further the accuracy of both methods in the assessment of $\mathrm{LV}$ volumes in clinical subjects and to verify the results of volume measurements in in vitro setting using phantoms.

\section{Methods}

\section{Phantoms}

Ten different cone-shaped phantoms were used in the study. Eight of them were manufactured with a symmetric geometric structure and two with asymmetric geometry that was considered to mimic LV with an aneurysm. All the phantoms were manufactured using water-based microbiology agar (1:30, agar:water, w/w) described by Burlew et al. [18]. In order to mimic blood-myocardium interface, a glass powder with very fine granularity was added to the outer compartment of the phantom considered to represent the cardiac walls $(1: 65$, glass powder:water-based agar, $\mathrm{w} / \mathrm{w}$ ), thus increasing the echocardiographic density. On the other hand, iodixanol (Visipaque, GE Healthcare, Little Chalfont, UK; $320 \mathrm{mg} \mathrm{I/}$ $\mathrm{ml}$ ) was added to the inner compartment of the phantom representing intracavital blood volume (1:20; iodixanol:waterbased agar, w/w) in order to increase the x-ray attenuation. The chosen concentration of iodixanol resulted in approximately the same Hounsfield values as in normal clinical scans. The specific weight of the mixture for the inner compartment was measured and the phantoms were weighed before and after adding the mixture. The exact volumes of the inner compartment of the phantoms (called phantom volumes) could then be calculated and ranged between 39 and $334 \mathrm{~mL}$ (Table 1).

The speed of ultrasound in the phantom material was measured in two pieces of the phantom material, one with and the other without glass powder, using piezoelectric crystals (one working as a transmitter and the other as a receiver) applied to the opposite sides of the test pieces. An electrical circuit and an oscilloscope connected to the crystals measured the time for a pulse of ultrasound to travel the distance between the crystals. The velocities of ultrasound within the phantoms were found to be 1470 $\mathrm{m} / \mathrm{s}$ in the outer section with the glass powder and 1540 $\mathrm{m} / \mathrm{s}$ in the inner section with iodixanol.

\section{Patients}

Nine patients (5 men), aged 64 (range 51-82) years were selected consecutively from a larger, prospective study evaluating efficiency of coronary MSCT for the detection of coronary artery disease in patients referred routinely for coronary angiography due to known or suspected coronary artery or valvular diseases. In all the selected patients, 3-D echocardiography was performed in addition to

Table I: True phantom volumes and the volumes obtained with simplified 3-D echocardiography and MSCT-CardIQ software.

\begin{tabular}{|c|c|c|c|}
\hline \multirow[t]{2}{*}{$\begin{array}{l}\text { Phantoms } \\
\text { (number) }\end{array}$} & \multirow[t]{2}{*}{$\begin{array}{l}\text { True volume } \\
\qquad(\mathrm{ml})\end{array}$} & \multicolumn{2}{|c|}{$\begin{array}{l}\text { Measured volume } \\
(\mathrm{ml})\end{array}$} \\
\hline & & $\begin{array}{l}\text { 3-D echo-cardiography } \\
\quad(\text { mean } \pm S D ; n=5)\end{array}$ & $\begin{array}{c}\text { MSCT-CardIQ software } \\
(\text { mean } \pm S D ; n=5)\end{array}$ \\
\hline 1 & 39 & $36.6 \pm 0.55$ & $40.4 \pm 1.14$ \\
\hline 2 & 98 & $99.4 \pm 0.55$ & $99.8 \pm 1.10$ \\
\hline 3 & 334 & $333.8 \pm 0.84$ & $348.4 \pm 5.64$ \\
\hline 4 & 293 & $291.6 \pm 1.14$ & $296.6 \pm 0.55$ \\
\hline 5 & 127 & $128.0 \pm 1.23$ & $131.6 \pm 1.68$ \\
\hline 6 & 52 & $51.6 \pm 0.55$ & $54.6 \pm 1.52$ \\
\hline 7 & 202 & $202.2 \pm 0.84$ & $209.2 \pm 1.64$ \\
\hline 8 & 246 & $246.0 \pm 1.23$ & $251.8 \pm 1.48$ \\
\hline 9 & 68 & $68.0 \pm 1.30$ & $72.6 \pm 0.55$ \\
\hline 10 & 157 & $158.6 \pm 3.44$ & $172.6 \pm 0.55$ \\
\hline
\end{tabular}

(I - 7: symmetric, and 9 - 10: asymmetric phantoms) 
MSCT. Both 3-D echocardiography and MSCT data were evaluated by independent trained interpreters. The study was approved by the local ethics committee of Karolinska University Hospital, Stockholm, Sweden.

\section{Simplified 3D-echocardiography}

The echocardiographic study was performed using a GE Vingmed Vivid 7 equipment and V3 matrix transducer allowing simultaneous acquisition of image data in 3 different planes positioned at $60^{\circ}$ angle to each other. In each of the 10 phantoms, the acquisition of echocardiographic data for the subsequent volume measurements was performed five times by placing the transducer close to the basal plane of the respective phantom submerged in water. In the patients, cineloops of 3 consecutive cardiac cycles were acquired at end-expiration from transthoracic apical window with a sampling frequency of 30 frames/s. The respective data sets were subsequently stored for offline analysis using a GE Echopac workstation and the Echopac software version BT06. In each of the three images generated simultaneously in three different planes by V3 Matrix transducer, the geometry and the area corresponding to LV cavity or to the inner section of the phantom (Fig. 1) was determined by manual border detection. Phantom volumes as well as human LV endsystolic and end-diastolic volumes were then estimated using Echopac software. LV ejection fraction was determined in human experiments as well.

\section{Multi-slice computed tomography}

The MSCT examinations of both patients and phantoms were performed employing a 64-slice spiral computed tomography scanner (General Electric (GE) LightSpeed VCT, Milwaukee, Wisc., USA). The gantry rotation time was 0.35 seconds, while a collimation of $64 \times 0.625 \mathrm{~mm}$ and a tube voltage of $120 \mathrm{kV}$ was used. In the patients, dose modulation was used and the tube current was diminished during systole resulting in the effective current of approximately 240-640 mAs and effective dose of 20.0 $\mathrm{mSv}$. All images (both in patients and phantoms) were

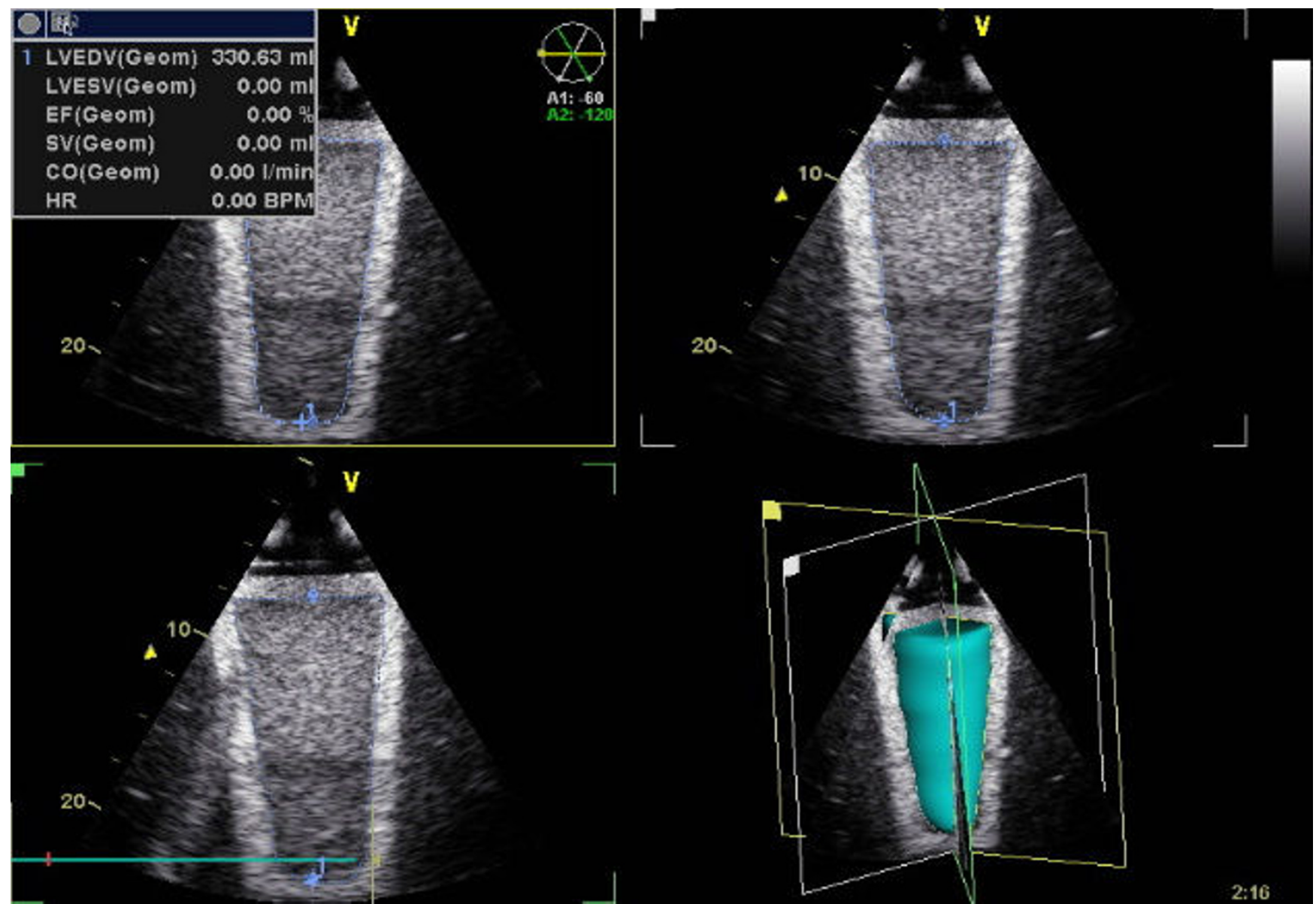

Figure I

Simplified 3D echocardiography. Example of the tracing of the inner contour of the phantom in 3 planes positioned at $60^{\circ}$ angle to each other and 3D reconstruction of phantom inner volume. 
acquired with slice thickness of 5 millimetres and spacing equal to 4 millimetres. A bolus injection of $85 \mathrm{ml}$ iodixanol (Visipaque, $320 \mathrm{mgI} / \mathrm{ml}$, GE Healthcare, Little Chalfont, UK) was given intravenously to the patients at a rate of $4-6 \mathrm{ml} / \mathrm{sec}$ followed by a flush with $50 \mathrm{ml}$ of saline. Using retrospective electrocardiographic gating, reconstruction of images from 10 phases of the cardiac cycle was done for the patients.

\section{MSCT analysis of ventricular function}

The patient and phantom CT data sets were subsequently transferred to a dedicated workstation (Advantage Workstation 4.1, GE Medical Systems, Milwaukee, Wisc., USA) for further processing using GE CardIQ Function Version 1.0.3 software. Volumes of the phantoms and the left ventricles were evaluated using this semi-automated software. Data sets consisting of $5 \mathrm{~mm}$ thick axial images were loaded into the CardIQ software. For the patients, the images were reconstructed with retrospective ECG gating at $10 \% \mathrm{R}-\mathrm{R}$ interval increments throughout the cardiac cycle. The diastolic volume was determined at $0 \%$ of the R-R interval and the systolic volume at $40 \%$ of the R-R interval. Short-axis images were reconstructed and automatic contour detection of the inner-volume of the phantoms and the myocardial border in left ventricle was performed (Fig. 2). Minor manual corrections were made of the myocardial borders only, and not of the phantom borders. Short axis images not including any part of the left ventricle or phantom were manually excluded prior to the volume calculations. At the level of the mitral valve, short axis images usually show both part of the left ventricle and an area in the middle delineated by the mitral valve leaflets. Only images with the ventricle representing more than a half of the image area in this region were cur- rently included. All manually selected images were processed further automatically and volumes were calculated. The papillary muscles were not automatically excluded from the calculated volume. The end-diastolic and endsystolic phases were selected and the volumes calculated. Five repeated volume measurements were performed in each phantom.

\section{Statistical analysis}

Univariate relations between echocardiographic and MSCT-CardIQ measured LV volumes as well as their relation to the true phantom volumes were tested with standard regression analysis. Assessment of agreement between 3-D echocardiography and MSCT-CardIQ software in volume measurements was performed using the method of Bland and Altman [18]. Paired data were compared using the Student's $t$-test. The data are presented as mean \pm SD unless otherwise stated.

\section{Results}

\section{Phantom experiments}

The used MSCT CardIQ software provided fully adequate delineation of the inner section of the symmetric phantoms (Fig. 2, right) but failed to detect sharp irregularities of the inner contour of the asymmetric phantoms and manual correction was therefore necessary. The results of phantom volume measurements correlated strongly with true phantom volumes, both for 3-D echocardiography $\left(\mathrm{r}^{2}=1.00 ; \mathrm{y}=0.51+1.00 \mathrm{x}\right)$ and MSCT-CardIQ software $\left(r^{2}=1.00 ; y=0.17+1.03 x\right)$. However, the results obtained with the two methods differed $(\mathrm{p}<0.001)$. The true phantom volumes and the average volumes based on five volume measurements in each phantom with each method are presented in Table 1, whereas Figure 3 (upper panel)

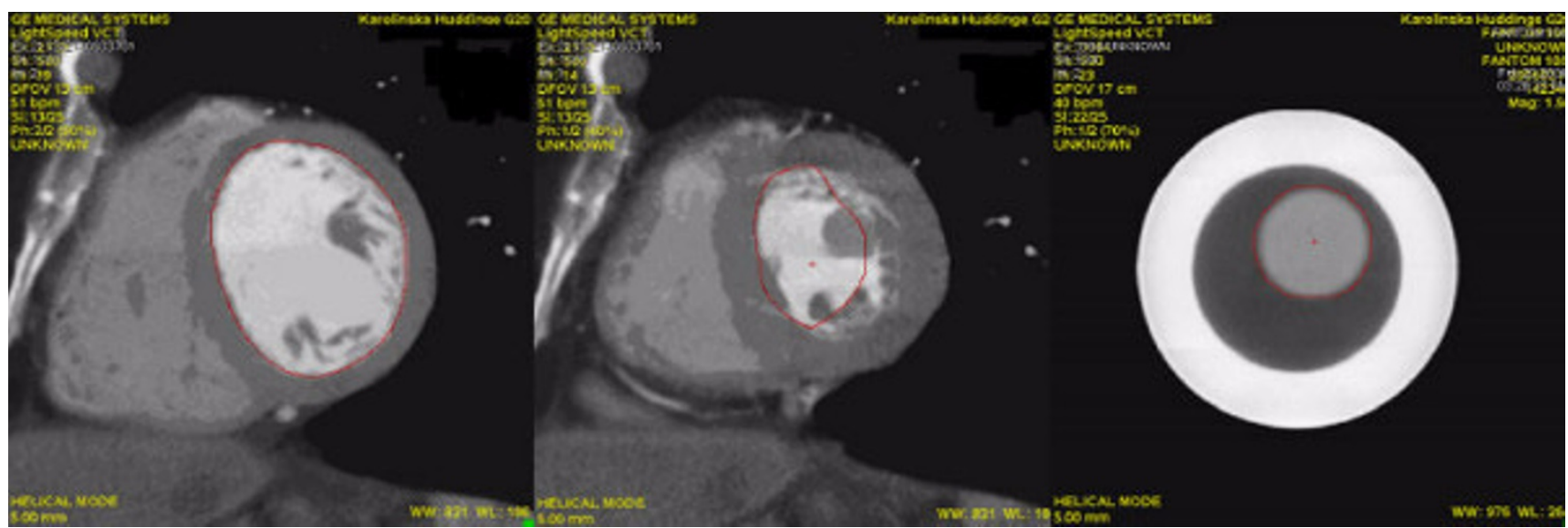

\section{Figure 2}

Automatic contour detection by MSCT. Examples of the automatic detection of the LV endocardial borders in shortaxis CT images in diastole ( $0 \%$ of the R-R interval; left), and systole ( $40 \%$ of the R-R interval; middle) and the detection of inner contour of a phantom (right) using GE CardIQ Function Version I.0.3 software. 

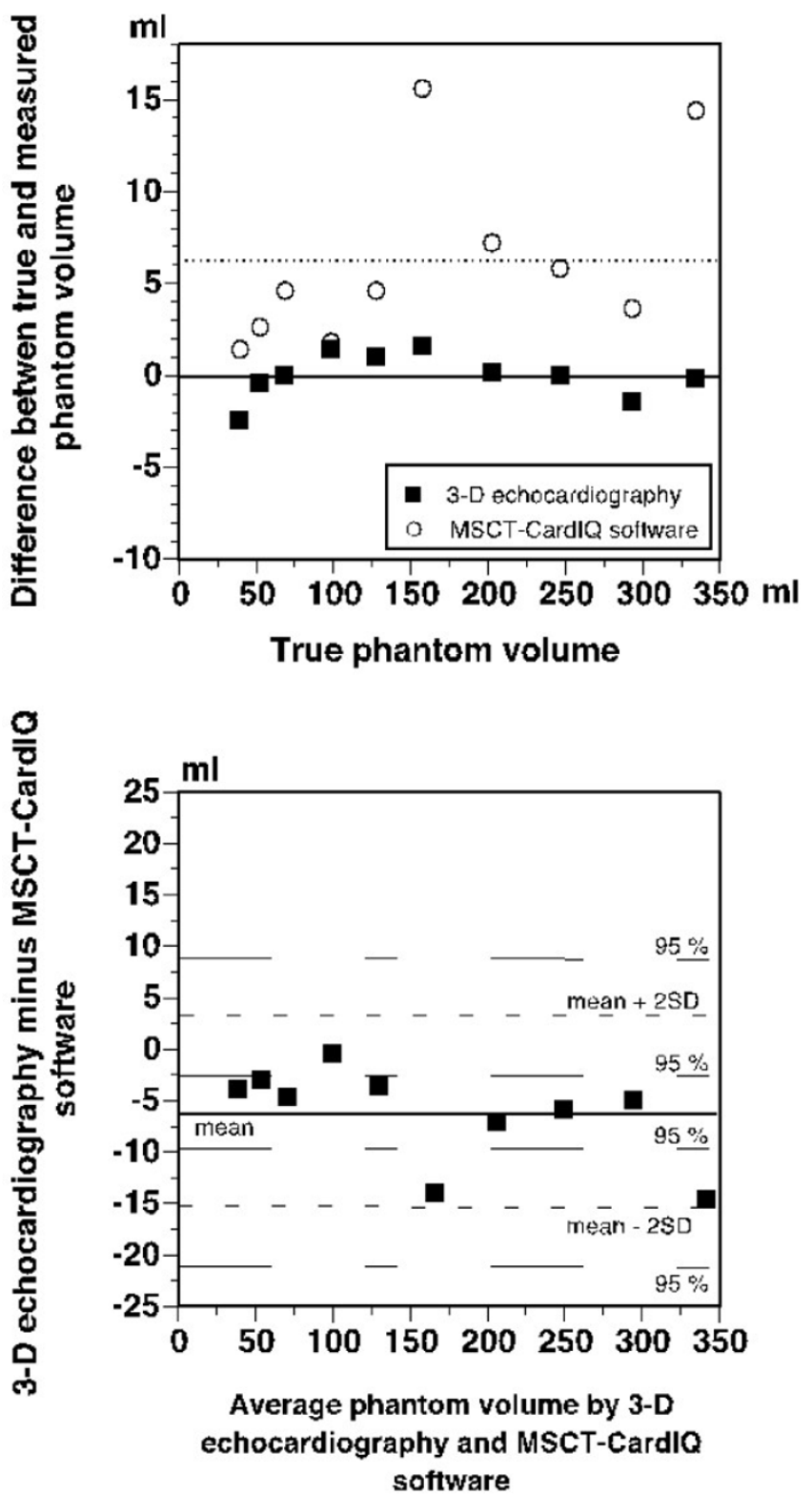

Figure 3

Differences between 3D-echocardiographic and MSCT-derived phantom volumes. Upper panel: Differences between true phantom volumes and the volumes measured by 3-D echocardiography and MSCT-CardIQ software. Mean differences for the respective methods are indicated. Lower panel: Bland-Altman plot of differences between phantom volumes measured by 3-D echocardiography and MSCT-CardIQ software against the average phantom volumes by the two methods. The mean difference and $95 \%$ confidence intervals for the mean difference \pm 2 SD are indicated.

displays the differences between true phantom volumes and the volumes measured with 3-D echocardiography and MSCT-CardIQ software. As can be seen, the results obtained with MSCT-CardIQ software overestimated the true phantom volumes in all cases and were more scattered than the results of 3-D echocardiographic measurements, resulting in a mean difference of $6 \pm 5 \mathrm{ml}$. The $95 \%$ confidence interval for the mean $\pm 2 \mathrm{SD}$ was +22 to $+10 \mathrm{ml}$ and +2 to $-10 \mathrm{ml}$, respectively. On the other hand, the $3-$ D echocardiographic results were distributed more evenly and closely to the true volumes (mean difference $0 \pm 1 \mathrm{ml}$ ) providing the true values in 2 cases and equal number of over- and underestimations for the rest of the phantoms. The 95\% confidence intervals for the mean difference \pm $2 \mathrm{SD}$ were +4 to $-1 \mathrm{ml}$ and +1 to $-4 \mathrm{ml}$, respectively.

The poor agreement between 3-D echocardiography and MSCT-CardIQ software is further illustrated in Bland-Altman plot in Figure 3 (lower panel). The consistent overestimation of the phantom volumes by MSCT-CardIQ software resulted in a mean difference between the results obtained with the both methods amounting to $-6 \pm 5 \mathrm{ml}$. The limits of agreement defined as mean difference $\pm 2 \mathrm{SD}$ were thus $+4 \mathrm{ml}$ and $-16 \mathrm{ml}$, respectively. The $95 \%$ confidence interval for the upper limit of agreement was +9 to $-3 \mathrm{ml}$ and the corresponding confidence interval for the lower limit of agreement was -10 to $-21 \mathrm{ml}$. This implies that the difference between the results of 3-D echocardiographic and MSCT-CardIQ software measurements may assume values ranging from $+9 \mathrm{ml}$ to $-21 \mathrm{ml}$.

\section{Measurements in patients}

Detection of the endocardial border and delineation of the LV cavity area in the patients by the MSCT-CardIQ software was less accurate than in the phantom experiments and required in most cases time consuming manual correction (Fig. 2, left and middle). A standard linear regression analysis revealed a moderate correlation between 3-D echocardiographic and MSCT-CardIQ software based volume measurements with $\mathrm{r}^{2}=0.62(\mathrm{y}=$ $10.99+1.19 \mathrm{x})$ for the end-systolic, and $\mathrm{r}^{2}=0.59(\mathrm{y}=$ $32.39+0.91 \mathrm{x})$ for the end-diastolic volume. Similar to what was observed in the phantom experiments, the results produced by the two tested methods (Table 2 ) differed significantly ( $p<0.05)$, except for LV ejection fraction.

The agreement between the results of 3-D echocardiographic and MSCT-CardIQ software based measurements of LV volumes in the patients was clearly poorer than was the case with the measurements of phantom volumes as evidenced by Bland-Altman analysis presented in Figure 4. As can be seen from the figure, a mean difference between the measurements performed with the two methods was $-23 \pm 40 \mathrm{ml}(-10 \pm 42 \mathrm{ml}$ for LV end-systolic and $-35 \pm 37 \mathrm{ml}$ for $\mathrm{LV}$ end-diastolic volume measurements). The limits of agreement (mean $\pm 2 \mathrm{SD}$ ) were $+57 \mathrm{ml}$ and $103 \mathrm{ml}$, respectively. The $95 \%$ confidence interval for the 
Table 2: Left ventricular volumes obtained in the same patient with 3-D echocardiography and MSCT-CardIQ software

\begin{tabular}{|c|c|c|c|c|}
\hline & & \multicolumn{2}{|c|}{ Measured volumes $(\mathrm{ml})$} & \multirow[t]{2}{*}{ Ejection fraction (\%) } \\
\hline & & Endsystolic & Enddiastolic & \\
\hline & Patient I & 30 & 62 & 52 \\
\hline & 2 & 31 & 89 & 65 \\
\hline & 3 & 36 & 99 & 64 \\
\hline \multirow{9}{*}{$\begin{array}{l}\text { S-D echo- } \\
\text { cardiography }\end{array}$} & 4 & 40 & 116 & 66 \\
\hline & 5 & 52 & 123 & 58 \\
\hline & 6 & 61 & 158 & 61 \\
\hline & 7 & 96 & 162 & 41 \\
\hline & 8 & 104 & 178 & 42 \\
\hline & 9 & 167 & 249 & 33 \\
\hline & Patient I & 40 & 145 & 72 \\
\hline & 2 & 41 & 137 & 70 \\
\hline & 3 & 36 & 116 & 69 \\
\hline MSCT-CardIQ & 4 & 72 & $14 \mid$ & 49 \\
\hline \multirow[t]{5}{*}{ software } & 5 & 73 & 167 & 56 \\
\hline & 6 & 61 & 164 & 63 \\
\hline & 7 & 32 & 162 & 80 \\
\hline & 8 & 91 & 170 & 46 \\
\hline & 9 & 263 & 347 & 24 \\
\hline
\end{tabular}

upper limit of agreement was $+93 \mathrm{ml}$ to $+23 \mathrm{ml}$ and the corresponding interval for the lower limit of agreement was $-69 \mathrm{ml}$ to $-138 \mathrm{ml}$, thus indicating that the difference
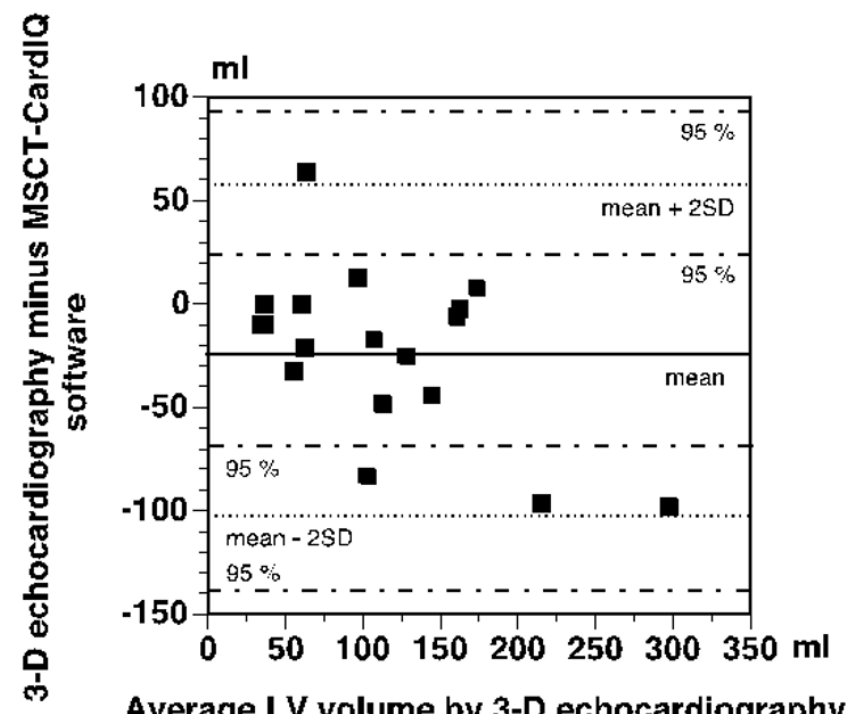

Average LV volume by $3-D$ echocardiography and MSCT-CardIQ software

\section{Figure 4}

Bland-Altman plot of differences between LV volumes measured by 3-D echocardiography and MSCT- seed growing software. The mean difference and $95 \%$ confidence intervals for the mean difference $\pm 2 \mathrm{SD}$ are indicated. Filled symbols - differences in end-systolic volume, open symbols - differences in end-diastolic volume. between the results obtained with the two tested methods may vary between $+93 \mathrm{ml}$ and $-138 \mathrm{ml}$.

\section{Discussion}

In the present study, the results of LV volume measurements by simplified 3-D echocardiography were compared with the results of 64-slice spiral computed tomography and the accuracy of the two methods was evaluated in two steps. First, both the methods were calibrated in vitro by comparison of the measured volumes with known volumes using phantoms, and second, the results of LV volume measurements with both the methods in patients were evaluated.

The phantoms used in the present experiments were especially designed to closely mimic the blood-myocardium border and to provide its equally good detection with both of the tested modalities. In order to fulfil these requirements, the speed of ultrasound wave travelling through the phantom should be in the range for which medical ultrasound equipment is calibrated for, i.e. around $1540 \mathrm{~m} / \mathrm{s}$, which is the speed of sound in most tissues imaged by echocardiography. The velocity of sound wave propagation through the currently employed phantoms was found to be $1470 \mathrm{~m} / \mathrm{s}$ in the section with the glass powder, and $1540 \mathrm{~m} / \mathrm{s}$ in the inner compartment of the phantom mimicking intracavital LV volume. The lower sound propagation velocity within the outer section of the phantoms resulted in a shift of depth coordinates for this section by $+0.0475 \mathrm{~mm}$ per each $\mathrm{mm}$. With the thickness of the phantom shell of approximately $8 \mathrm{~mm}$, the total shift in its depth positioning would be about 0.4 
$\mathrm{mm}$ and this would certainly introduce a systematic error if the total phantom volume was measured. However, since the velocity of ultrasound within the inner section of the phantoms was equal to the calibration velocity for the used equipment, imaging of the phantom "cavity" was not distorted and the currently performed calculations of the inner phantom volumes were therefore not biased.

The absolute value of the $\mathrm{x}$-ray attenuation by the phantom structures was not as critical for the accuracy of the measurements as the velocity of sound wave propagation, but a proper automated border detection by the software required occurrence of a significant attenuation difference between the sections mimicking myocardium and LV cavity. In the present experiments, the chosen concentration of iodixanol in the inner section of the phantoms resulted in the same Hounsfield values as those obtained in routine diagnostic images and the delineation of this section was fully adequate in the case of the symmetric phantoms. The delineation of the "cavities" of the asymmetric phantoms was not entirely satisfactory and sharp contour irregularities of the inner section were not detected resulting in a tendency to overestimation of the phantom "cavity" volume that necessitated manual correction. These inadequacies were, however, not caused by insufficient attenuation differences but by software-dependent limitations to handle highly irregular borders.

The results of phantom volume measurements by simplified 3-D echocardiography showed very good agreement with the true phantom volumes resulting in mean difference between the respective data close to zero with a possible overestimation or underestimation of true volumes by $4 \mathrm{ml}$ that is fully acceptable in clinical practice. On the other hand, the results produced by MSCT-CardIQ software were significantly more biased with a possibility of overestimation by $22 \mathrm{ml}$ or underestimation by $10 \mathrm{ml}$. Despite the fact that there was a strong relationship between the true phantom volumes and the volumes measured with MSCT-CardIQ software, considerable discrepancies might occur between the true and measured values, and consequently, also between the results obtained with the both methods, thus limiting their interchangeable clinical use.

The poor agreement between the results of 3-D echocardiography and those generated by MSCT-CardIQ software was particularly striking when $\mathrm{LV}$ volume measurements in patients were compared. Several previous studies in which assessment of LV volumes with MSCT technique and 2-D echocardiography was evaluated produced results showing a good agreement between these two methods $[4-7,19]$. In addition, a good correlation was found between MSCT measurements, and between 3-D echocardiography and magnetic resonance imaging [2-
$4,8,9,11,14,17]$. Even if the methodological details and the MSCT software used in the above-mentioned studies differed from the present approach, a similar good relationship with acceptable volume assessments could still be expected to exist between MSCT and 3-D echocardiography. However, the volume overestimation by MSCTCardIQ software in relation to 3-D echocardiography was found to be considerable in clinical situation and the mean difference between the results obtained with two methods increased from moderate $-6.2 \mathrm{ml}$ in phantom experiments to $-22.6 \mathrm{ml}$ in patient measurements. At the same time the variation of the results increased considerably resulting in unacceptable limits of agreement ranging between $+92.9 \mathrm{ml}$ and $-138.1 \mathrm{ml}$, thereby somewhat in disagreement with some of the above-mentioned earlier published data. On the other hand, the present results showing the larger volumes obtained by MSCT than those measured by 3-D echocardiography are in keeping with the results of recently published study of Sugeng et al. [17], in which MSCT was found to produce significant overestimation of $\mathrm{LV}$ volumes measured by magnetic resonance whereas real-time 3-D echocardiography compared more favourably in this respect.

When considering the results of 3-D echocardiographic and MSCT-CardIQ software based measurements of LV volumes, it has to be kept in mind that the methodological error may be greater in clinical setting than in in vitro phantom measurements. The simplified 3-D echocardiographic technique relies on a computation of LV volume by interpolation of manually traced endocardial border from three different 2-D planes. Consequently, any possible local changes in LV geometry between the traced planes will remain undetected, resulting in calculation error. On the other hand, the delineation of LV area contour with MSCT software may underestimate or overestimate the contribution of the contrast filled crevices and small cavities of the LV myocardial trabecular network. Multiplied by slice thickness, these possible area errors would inevitably result in erroneous LV volume estimations. In addition, the relatively low temporal resolution of the method and the nature of the used MSCT software calculating $\mathrm{LV}$ volume by integrating data from short-axis slices make the determination of volumes near to mitral annulus uncertain. However, the discrepancy between the 3-D echocardiographic and MSCT-CardIQ software generated LV volumes in the present study was substantial and suggests the existence of other colluding sources of errors as well. Since the MSCT software performed rather unsatisfactory on phantoms as well, it appears reasonable to believe that the observed considerable differences between the two methods might have been caused by additional, possibly MSCT algorithm dependent error. If so, the present results cast doubt on diagnostic applicability of the currently tested MSCT approach. 
As an additional comment to the present results, it is perhaps worthwhile to mention that after completion of this study, we also compared simplified 3-D echocardiography with another MSCT software (GE Software Ejection Fraction). This was a seed growing software that instead of outlining the endocardial border, worked by binding voxels with similar Hounsfield values assumed to originate from the same sort of tissue. Since the software did not work satisfactorily in phantoms, only measurements of LV volumes in patients were performed. The results are presented in Bland-Altman plot in Figure 5 and show that the agreement between this MSCT software and 3-D echocardiography was poor as well and clearly not better than that with MSCT-CardIQ software (mean difference \pm SD equal to $-11 \pm 43 \mathrm{ml}$ and limits of agreement 75 and -97 $\mathrm{ml})$. The $95 \%$ confidence intervals for mean \pm 2 SD (112 to $39 \mathrm{ml}$ and from -60 to -134$)$ indicated that the difference between the methods might vary to the same considerable extent as for MSCT-CardIQ software.

In this context, it should be remembered that beside the currently reported results of volume measurements, there are other important factors that would favour the diagnostic use of 3-D echocardiography. For example, the effective radiation dose associated with the MSCT procedure employed in the present study was $20.0 \mathrm{mSv}$ that is a dose

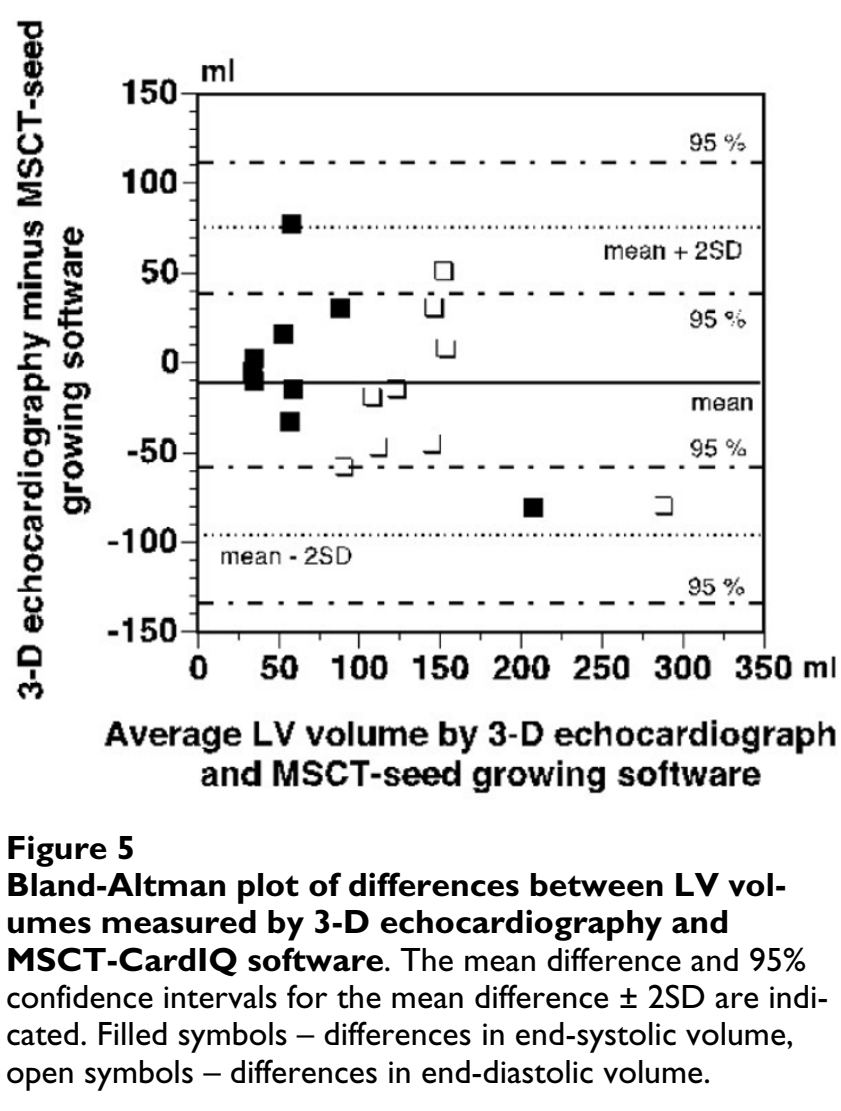

equivalent to 1000 chest x-rays or nearly 7 years of background radiation [20]. This dose results in an extra risk of long-term cancer development of about 1/1000 exposed individuals and the procedure is at the same time about 3 times more expensive that conventional echocardiography [21]. On the other side, the 3-D echocardiographic approach, in addition to being radiation-free and more cost-effective, does not require the use of nephrotoxic contrast media, and is faster and easier to perform. These facts should be taken into consideration when adopting As Low As Reasonably Achievable (ALARA) philosophy when performing diagnostic cardiac imaging.

\section{Conclusion}

The present results demonstrate that simplified 3-D echocardiography provides a reliable and significantly more accurate assessment of phantom volumes than MSCT-CardIQ software. The discrepancy between the results of both methods increase considerably when LV volume is measured and the limits of agreement are not acceptable for interchangeable diagnostic use of the both methods. Judging from its performance on phantoms, simplified 3-D echocardiography can be expected to provide most accurate $\mathrm{LV}$ volume assessments in clinical situations.

\section{Competing interests}

The authors declare that they have no competing interests.

\section{Authors' contributions}

MM constructed the phantoms, performed phantom volume measurements with 3-D echo and MSCT, and prepared the manuscript. RW performed the 3-D echo examination and LV volume calculation on patients. KC, JR and HMA performed MSCT examinations on both phantoms and patients. They performed volume calculations in patients with both software and participated in the manuscript preparation. JN performed statistical analysis and was responsible for the final layout of the manuscript. LÅB initiated and supervised the study and participated in the manuscript preparation. All authors read and approved the manuscript.

\section{Acknowledgements}

This study was supported by grants from the Swedish Heart-Lung Foundation.

\section{References}

I. Hosoi S, Mochizuki T, Miyagawa M, Shen Y, Murase K, Ikezoe J: Assessment of left ventricular volumes using multi-detector row computed tomography (MDCT): Phantom and human studies. Radiation Medicine 2003, 2 1:62-67.

2. Heuschmid M, Rothfuss JK, Schroeder S, Fenchel M, Stauder N, Burgstahler C, Franow A, Kuzo RS, Kuettner A, Miller S, Claussen CD, Kopp AF: Assessment of left ventricular myocardial function using 16-slice multidetector-row computed tomography: comparison with magnetic resonance imaging and echocardiography. Eur Radiol 2005, 16:55I-559. 
3. Raman SV, Shah M, McCarthy B, Garcia A, Ferketich AK: Multidetector row cardiac computed tomography accurately quantifies right and left ventricular size and function compared with cardiac magnetic resonance. Am Heart J 2006, I5 1:736-744.

4. Yamamuro M, Tadamura E, Kubo S, Toyoda $H$, Nishina T, Ohba M, Hosokawa R, Kimura T, Tamaki N, Komeda M, Kita T, Konishi J: Cardiac functional analysis with multi-detector row $C T$ and segmental reconstruction algorithm: comparison with echocardiography, SPECT, and MR imaging. Radiology 2005, 234:38I-390.

5. Henneman MM, Bax JJ, Schuiif JD, Jukema JW, Holman ER, Stokkel MPM, Lamb HJ, de Roos A, van der Wall EE: Global and regional left ventricular function: a comparison between gated SPECT, 2D echocardiography and multi-slice computed tomography. Eur J Nuc Med Mol Imaging 2006, 33:| $452-1460$.

6. Salm LP, Schuijf JD, de Roos A, Lamb HJ, Vliegen HW, Jukema JW, Joemai $R$, van der Wall EE, Bax JJ: Global and regional function assessment with 16-detector row CT: Comparison with echocardiography and cardiovascular magnetic resonance. Eur J Echocardiography 2006, 7:308-3I4.

7. Schuijf JD, Bax JJ, JWJukema, Lamb HL, Salm LP, de Roos A, van der Wall EE: Assessment of left ventricular volumes and ejection fraction with 16-slice multi-slice computed tomography; comparison with 2D-echocardiography. Intl J Cardiology 2007, I | 6:201-205.

8. Buck T HP Wentz KU, Tkalec W, Nesser HJ, Erbel R: Tomographic three-dimensional echocardiographic determination of chamber size and systolic function in patients with left ventricular aneurysm:comparison to magnetic resonance imaging, cineventriculography, and two-dimensional echocardiography. Circulation 1997, 96:4286-4297.

9. Caiani EG, Corsi C, Zamorano J, Sugeng L, MacEneaney P, Weinert L, Battani R, Gutierrez JL, Koch R, Perez de Isla L, Mor-Avi V, Lang RM: Improved semiautomated quantification of left ventricular volumes and ejection fraction using 3-dimensional echocardiography with a full matrix-array transducer: comparison with magnetic resonance imaging. J Am Soc Echocardiogr 2005, 18:779-788.

10. Gopal AS, Keller AM, Rigling R, King Jr DL, King DL: Left ventricular volume and endocardial surface area by three-dimensional echocardiography: Comparison with two-dimensional echocardiography and nuclear magnetic resonance imaging in normal subjects. J Am Coll Cardiol 1993, 22:258-270.

II. Gutiérrez-Chico JL, Zamorano JL, Pérez de Isla L, Orejas M, Almeria C, Rodrigo JL, Ferreirós J, Serra V, Macaya C: Comparison of left ventricular volumes and ejection fractions measured by three-dimensional echocardiography versus by two-dimensional echocardiography and cardiac magnetic resonance in patients with various cardiomyopathies. Am J Cardiol 2005, 95:809-8I3.

12. Sapin PM, Schröder KM, Gopal AS, Smith MD, DeMaria AN, King DL: Comparison of two- and three-dimensional echocardiography with cineventriculography for measurement of left ventricular volume in patients. J Am Coll Cardiol 1994, 24: I054- 1063.

13. Schröder KM, Sapin PM, King DL, Smith MD, DeMaria AN: Threedimensional echocardiographic volume computation: in vitro comparison to standard two-dimensional echocardiography. J Am Soc Echocardiogr 1993, 6:467-475.

14. Chan J, Jenkins C, Khafagi F, Du L, Marwick TH: What is the optimal clinical technique for measurement of left ventricular volume after myocardial infarction? A comparative study of 3-dimensional echocardiography, single photon emission computed tomography, and cardiac magnetic resonance imaging. J Am Soc Echocardiogr 2005, 19:192-201.

15. Krenning B], Voormolen MM, van Geuns RJ, Vletter WB, Lancée CT, de Jong N, Ten Cate FJ, van der Steen AFW, Roelandt JRTC: Rapid and Accurate Measurement of Left Ventricular Function with a New Second-Harmonic Fast-Rotating Transducer and Semi-Automated Border Detection. Echocardiography 2006, 23:447-454.

16. Mannaerts HFJ, van der Heide JA, Kamp O, Papavassiliu T, Marcus JT, Beek A, van Rossum AC, Twisk J, Visser CA: Quantification of left ventricular volumes and ejection fraction using freehand transthoracic three-dimensional echocardiography: Com- parison with magnetic resonance imaging. J Am Soc Echocardiogr 2003, 16:101-109.

17. Sugeng L, Mor-Avi V, Weinert L, Niel J, Ebner C, SteringerMascherbauer R, Schmidt F, Galuschky C, Schummers G, Lang RM, Nesser HJ: Quantitative assessment of left ventrricular size and function. Side-by-side comparison of realt-time threedimensional echocardiography and computed tomography with magnetic resonance reference. Circulation 2006, I | 4:654-66|.

18. Altman DG, Bland JM: Statistical methods for assessing agreement between two methods of clinical measurement. Lancet 1986, 32:307-317.

19. Dirksen MS, Jukema JW, Bax JJ, Lamb HJ, Boersma E, Tuinenburg JC, Geleijns J, van der Wall EE, de Roos A: Cardiac multidetectorrow computed tomography in patients with unstable angina. Am J Cardiol 2005, 95:457-46I.

20. Einstein AJ, Moser KW, Thompson RC, Cerqueira MD, Henzlova MJ: Radiation Dose to Patients From Cardiac Diagnostic Imaging. Circulation 2007, I I6: 1290-1305.

21. Picano E: Economic and biological costs of cardiac imaging. Cardiovascular Ultrasound 2005, 3:13.
Publish with Bio Med Central and every scientist can read your work free of charge

"BioMed Central will be the most significant development for disseminating the results of biomedical research in our lifetime. "

Sir Paul Nurse, Cancer Research UK

Your research papers will be:

- available free of charge to the entire biomedical community

- peer reviewed and published immediately upon acceptance

- cited in PubMed and archived on PubMed Central

- yours - you keep the copyright
BioMedcentral 